\title{
21. LIGHT OF THE NIGHT SKY (LUMIERE DU CIEL NOCTURNE)
}

\author{
President : A.C. Levasseur-Regourd \\ Vice President : M.S. Hanner \\ Organizing Committee : \\ S. Bowyer, R. Dumont, Y. Galperin, J. Houck, P. Lamy, C. Leinert, K. Mattila, T. Mukai
}

\section{INTRODUCTION}

The different components of the light of the night sky are integrated along the line of sight of the observer located on planet Earth or on the near Earth's environment. They encompass a huge scale of distances, ranging from hundreds of kilometers in the Earth's atmosphere (airglow lines, bands and continuum), to astronomical units in the interplanetary medium (zodiacal light continuum), thousand of light years in the interstellar medium (diffuse galactic light and integrated starlight) and even much more in the extragalactic universe (extragalactic background radiation).

The problems encountered in distinguishing the individual components of the night sky, as perfectly described by Mattila in the preface of the proceedings of IAU symposium 139 (Bowyer and Leinert eds., Kluwer, Dordrecht, 1990) are similar to "the attempt to restore a fresco...; one has to remove the more recent layers... with painstaking care... ; our task in disentangling the several components of the light of the night sky is even more difficult, because we want to restore each one of the different layers of the sky as an individual masterpiece of nature..., the only exception... (being) the artificial light component produced by humanity itself".

From the point of view of commission 21 , the analysis of the night sky components is made from the ultraviolet to the infrared domains. Very similar problems are indeed encountered in ultraviolet and infrared, as in the more traditional optical domain; the physical sources of each of the various components of the night sky are quite comparable all over this spectrum ; and the techniques of data analysis developed in one of these spectral domains are rather directly applicable to the others.

During the triennium covered by this report, two IAU meetings have been organized by commission 21, providing an up-to-date presentation of our knowledge on interplanetary dust and zodiacal light (IAU Coll. 126, see III), and on galactic and extragalactic background radiation (IAU Symp. 139, see IV). The two corresponding proceedings are extensively quoted in these pages, prepared with the help of many commission members, who kindly provided information on their recent research.

To make the presentation of the references concise, we have used the following abbreviations :

A \& A AdvSpaRes

AJ

AnnRev A \& A

ApJ

PlaSpaSci

QJRAS

SpaSci Rev
Astron. Astrophys.

Advances in Space Research.

Astron. J.

Ann. Review of Astron. Astrophys.

Astrophys. J.

Planetary Space Sci.

Quaterly J. of the R.A.S.

Space Sci. Review.
OEID

GEBR

IAU Coll. 126

Origin and evolution of interplanetary dust A.C. Levasseur-Regourd ed. Kluwer, Tokyo, 1991

IAU Symp. 139

The galactic and extragalactic background radiation S. Bowyer and C. Leinert eds Kluwer, Dordrecht, 1990 


\section{AIRGLOW}

As mentioned in previous triannual reports, the numerous papers about aeronomic problems related to airglow (e.g. winds in the upper atmosphere, laboratory data) are rather likely to be included in the category of geophysics. We shall typically not detail the works about the stratospheric $\mathrm{O}_{3}$ (see for instance, for aeronomic chemistry of $\mathrm{O}_{3}$, Nicolet, PlaSpaSci, 37, 12, 1621, 1989). However, of major interest to astronomers are the airglow studies which provide information on the light pollution or on the fluctuation of the nightglow.

Broad band measurements of the night sky brightness have been performed from various observatory sites, with emphasis on the problems of light pollution (Angione, Garstang). The programme of airglow survey, started in 1979 at Kiso, Japan, with a newly designed photometric system, has been continued. Measurements of zenith intensities [in $S_{10}(v)$ ] of the 555.7,589, 589.6 and $630 \mathrm{~nm}$ airglow lines are made on every moonless clear nights, together with measurements of the background continuum at $530 \mathrm{~nm}$. Two volumes of airglow data plots (1979-1988) have now been published by Tanabe, Tanaka, Miyashita and Takechi from the world data center $\mathrm{C}_{2}$ for airglow.

Spectroscopic problems associated with dayglow have been treated by Chamberlain (Icarus, $70,476,1987)$. The asymmetries to be expected from the fine structure splitting of the $\mathrm{H}$ levels are compared with the interplanetary and interstellar background ; a simplified method of computation of polarization and anisotropy of emissions arising from resonant or fluorescent scattering is presented (Chamberlain, Icarus, 84, 106, 1990). Absolute transition probabilities have been given for the various bands systems of molecular oxygen; the nature of the precursors in the nightglow OI green line has been discussed (Bates, PlaSpaSci, 36, 9, 869, 875 and 883, 1988).

\section{ZODIACAL LIGHT}

At the end of the triennium covered by this report, the IAU colloquium 126 "Origin and evolution of interplanetary dust", here referred to as OEID, was organized by commission 21 . A significant number of contributions about zodiacal light can be found in the proceedings which are to be published in 1991 by Kluwer, Tokyo (A.C. Levasseur-Regourd ed., T. Mukai sec.). An extensive review about this topic can also be found in the chapter "Interplanetary dust" of "Physics of the inner heliosphere" (R. Schwenn and E. Marsch eds.), which was written in 1988 by C. Leinert and E. Grün. Due to numerous efforts in the interpretation of infrared zodiacal light observations, major progresses have been made in the area of the optical properties of the interplanetary dust grains.

\section{Zodiacal light observations}

In the ultraviolet and visible domains, zodiacal light is due to solar light scattered on interplanetary grains. No new major observations have been obtained since the previous report. In the visible domain, there is now a fair agreement on the absolute photometry and polarization, at least for elongations greater than $30^{\circ}$, and on the localization of the near ecliptic symmetry surface. The questions of the stability of the zodiacal light (Leinert and Pitz, A \& A, 210,399, 1989) and of the increase in intensity at small wavelengths (Lillie, OEID) are still discussed.

Murthy at al. (A \& A, 231, 187, 1990) have derived the zodiacal light contribution from spectrometric observations aboard the space shuttle in the $165-310 \mathrm{~nm}$ range. They found in almost linear increase, up to a factor of 2 , in the color with ecliptic latitude ; they concluded that the small grains responsible for the ultraviolet scattering have a much broader distribution with distance from the ecliptic plane than do the larger grains responsible for the visible scattering. 
With the occurrence of the 1991 solar eclipse and the preparation of the 1995 SOHO mission (Koutchmy, SpaSciRev, 47, 95, 1988 and ESA SP 1104, 1989 ; Lamy OEID), new observations are to be expected towards the F-corona and the inner zodiacal cloud, where a sublimation zone could be suspected (Mann, OEID ; Levasseur-Regourd et al., OEID). It is also likely that new observations, relatively easy to deconvolve, will be obtained towards the antisolar-Gegenschein region (Hong and Kwon, OEID).

In the infrared domain, zodiacal light is due to the thermal emission from interplanetary dust grains of both cometary and asteroidal origin (Sykes, OEID). It is indeed the strongest source of diffuse radiation in the night sky at wavelengths 5-50 $\mu \mathrm{m}$. In 1983, the IRAS spacecraft carried out a 10 month survey at $12,25,50$ and $100 \mu \mathrm{m}$ for solar elongations $60^{\circ}-120^{\circ}$, and these data are still being analysed. The data are available on the IRAS observations history file (Beichmann, AnnRev A \& A, 25, 521, 1987 ; Boulanger and Perault, ApJ, 330, 964, 1988 ; Hauser, in "Comets to cosmology", A. Lawrence ed., 27, 1988). The calibration discrepancy of about a factor of 1.5 versus previous rocket experiments should be resolved by DIRBE experiment (Mather et al., ApJ, 354, L37, 1990).

The Cosmic Background Explorer (COBE) spacecraft has recently completed a survey of the diffuse sky background at infrared and millimeter wavelengths. The DIRBE experiment allowed the sky to be mapped simultaneously in 10 bandpasses from 1 to $300 \mathrm{~m}$, for solar elongations between $64^{\circ}$ and $124^{\circ}$. Very accurate measurements of the diffuse zodiacal "foreground" should therefore appear in a near future ( Hauser private communication; Hanner, OEID).

\section{Interpretation of the observations}

The observed zodiacal light, whether viewed in ultraviolet, visible or infrared light, is an integral over the line of sight. The spatial distribution (density) and the scattering or emitting properties of the dust are convolved together in this integration. The situation is somehow more intricate for scattered solar light, since the scattering is a function of the phase angle, than for the thermal emission, which can be assumed to be isotropic.

Various models have been developed to fit the infrared observations (e.g. Deul and Wolsencroft, A \& A, 196, 277, 1988 ; Good, AdvSpaRes, 6, 83, 1987 ; Reach and Heiles, OEID). Also inversion methods have been developed to recover the density and the local optical properties of the interplanetary dust. Hovenier and Bosma (OEID) have estimated that the mean scattering cross section of the dust grains vanishes beyond $\approx 3.7$ AU. Hong and Um (ApJ, 320, 928, 1987) have concluded that the density $\mathrm{x}$ cross section cannot be represented by a single gradient in the symmetry plane, and that the infrared absorption cross section varies with solar distance in a manner requiring more than one dust component.

From their nodes of lesser uncertainty method, Dumont and Levasseur-Regourd (A \& A, 191, 154,1988 ; AdvSpaRes, 6, 7, 87, 1987) have computed the heliocentric gradient of local polarization degree (at $90^{\circ}$ phase and near $1 \mathrm{AU}$ ) to be of the order of 0.5 , and the gradient of local bulk albedo to be about - 0.3 . The density gradient, equal to the difference between that of the local brightness $(-1.3)$ and of the local albedo, is indeed of the order of - 1. Also, out of the symmetry plane, the local polarization degree (at constant phase and solar distance) and the local temperature are found to decrease, while the local bulk albedo is found to increase, as the grains' orbits are more inclined upon the symmetry plane (Levasseur-Regourd et al., AdvSpaRes, 10, 3, 163, 1990 ; Renard et al., OEID).

The heterogeneity of the zodiacal cloud becomes increasingly evident, with a probable mixture of two populations of grains. One population (low albedo, high polarization) is concentrated near the symmetry plane and could have been produced by periodic comets and/or asteroids ; the other one (higher albedo, lower polarization) is less flattened and could originate in new comets or betameteoroids (Levasseur-Regound et al., OEID). 
The discrepancy between the models derived from visual observation fittings, which require a solar bulge in the density distribution, and the models derived from infrared observations fittings, which rather require a solar hole, is indeed solved with a bimodal population, one component having is maximum close to the symmetry plane, the other one being isotropically distributed (Giese and Kneissel, Icarus, 81, 369, 1989 ; Kneissel and Mann, OEID).

\section{Light scattering by dust particles}

A better understanding of the scattering by dust particles has been achieved thanks to both laboratory measurements and theoretical works. The groups at Bochum, Gainesville and Marseilles (e.g. Killinger, Zerull, Gustavson, Bliek, Lamy) have been very active in promising experimental work. The field of theoretical investigations has been most active in Helsinki (Muinonen, thesis ; Muinonen and Lumme, OEID), Leiden (Stammes, thesis), and Marseilles (Lamy and Perrin, Icarus, $76,100,1988$; AdvSpaRes, 9, 3, 241, 1989), with studies of the interaction of light with rough and porous grains, using the discrete dipole approximation or the eikonal approximation.

The colorimetric properties of a dust cloud, with implications for the zodiacal light, have also been studied (Perrin and Lamy, A \& A, 226, 288, 1989). The size distribution of interplanetary dust has been compared to that of cometary dust (Mukai, in "Highlights in astronomy", McNally ed., 8, $305,1989)$.

The phase dependence of the scattering function and of the local polarization has been compared to that of cometary dust (Levasseur-Regourd et al., Icarus, 86, 264, 1990). It is likely (Mukai et al., Icarus, 80, 254, 1989 and in "Evolution of interstellar dust", A. Bonetti et al. eds., 397,1989 ; Lamy, OEID) that the heliocentric changes in polarization and albedo previously mentioned are connected with a change in the size distribution of the dust grains, resulting from collisions and loss of absorbing organic material.

\section{GALACTIC AND EXTRAGALACTIC COMPONENTS}

The study of the diffuse astronomical background has taken new dimensions with the discovery of diffuse backgrounds in the $\gamma$-ray and $X$-ray bands and the precise observations in the microwave spectrum of the $\approx 3 \mathrm{~K}$ cosmic background. In the ultraviolet, visible and infrared domains also, new data have been acquired. The first international symposium on these topics, conceived by K. Mattila (past president of commission 21), and sponsored by IAU (Symposium 139, "The galactic and extragalactic background radiation", here referred to as GEBR) has taken place in 1989. All of the about 100 contributions which can be found in the proceedings of this most successful conference ( $S$. Bowyer and C. Leinert eds., Kluwer, Dordrecht) could be quoted in a review about the progresses in our knowledge of night sky galactic and extragalactic components ! Indeed, as summarized by Puget (GEBR, 485), the study of the background radiation requires absolute measurements of low brightness over very extended regions; data at all frequencies can now be compared ; in the ultraviolet to submillimetric range, they give useful constraints on the astrophysical interpretation of the data.

\section{Ultraviolet background}

The diffuse extreme ultraviolet background $(\approx 10 \rightarrow 100 \mathrm{~nm}$ ) has been studied by the Berkeley group from an EUV spectrometer flown on a sounding rocket (Labov, App. Optics, 28, 5073, 1989). Statistically significant features are found near 10,20,30.4, 58.4, 60.8 and $63 \mathrm{~nm}$. Some of them are produced by solar flux scattering from neutral or ionized He ; features at 10 and $63 \mathrm{~nm}$ are likely to be produced by hot $\left(10^{5}\right.$ to $\left.10^{6} \mathrm{~K}\right)$ gas in the local interstellar medium (Bowyer et al., COSPAR 1990). 
Significant advances in the study of the far ultraviolet background $(\approx 100 \rightarrow 200 \mathrm{~nm})$ have been made with a number of sounding rocket and shuttle-borne experiments (Bowyer, GEBR, 171). The ultraviolet UVX experiment on the space shuttle consisted of two instruments, from John Hopkins and Berkeley, which were aligned and operated simultaneously (Henry et al., GEBR, 227 ; Hurwitz et al., GEBR, 229).

The first instrument's observations are consistent with a spectrally flat background between 130 and $170 \mathrm{~nm}$, with intensities of 200 to 700 photons $\mathrm{cm}^{-2} \mathrm{~s}^{-1} \mathrm{sr}^{-1} \AA^{-1}$. There are some evidences for spatial variations, with no indication of large variations (Tennyson at al., ApJ, 330, 445, 1998 ; Murthy et al., ApJ, 336, 954, 1989).

The second instrument's observations confirm that most of the far ultraviolet background is galactic in origin, and strongly suggest that the dust grains which scatter in ultraviolet are different from those which scatter in visible, since they have a low albedo (0.15 to 0.2$)$ and scatter almost is isotropically (Martin and Bowyer, ApJ, 383, 677, 1989 ; Martin et al. GEBR, 223).

\section{Optical Background}

Optical observations of galactic light have been reviewed by Toller (GEBR, 21). Modern star count methods and stellar photometry measurements have been developed, respectively by Tanabe and Mori (GEBR, 103), and Leinert and Thiele (GEBR, 105), together with space-based photometric surveys. Integrated starlight is the dominant component. The contribution of diffuse galactic light in the interstellar medium peaks slightly off the galactic plane and declines towards higher latitudes (Toller et al., A \& A, 188, 24, 1987).

The separation of the diffuse galactic light, which in the ultraviolet and optical domains results from the scattering of galactic starlight by interstellar dust grains, has been reviewed by Witt (GEBR, 127). In the optical domain, reasonably consistent scattering properties have been derived; there is a reasonably close agreement on a value of $\approx 0.6$ for the dust albedo and on a phase function asymmetry factor of about 0.7 , with strongly forward scattering grains.

Measurements of the polarization of galactic background light in the visible spectral region suggest a linear polarization of a few percent, perpendicular to the galactic plane ; despite the difficulties of both measurement and interpretation, the polarization may be expected to provide new information on the galactic structure (Leinert, GEBR, 75).

\section{Infrared galactic background}

Results obtained with IRAS satellite at high galactic latitude have been reviewed by Boulanger (GERB, 139). Numerous contributions can be found in "Comets to cosmology", A. Lawrence ed., Springer-Verlag, Berlin 1988. The galactic emission has a complex morphology with filamentary structures, so-called the IR cirrus. The infrared emission seems to arise mostly from dust associated with neutral atomic gas. The upper limit of the intensity of the far infrared background is still difficult to obtain, since the brightness of the extragalactic sky is masked by zodiacal light and high latitude cirrus (Longair, GEBR, 469 ; Habing, 81, Puget, 113, Désert et al., 130, in "Comets to cosmology").

\section{Extragalactic background}

Deep CCD imaging surveys of the extragalactic background have been carried out up to mag 29 (Tyson, GEBR 245 ; AJ, 96, 1, 1988). They reveal an isotropic population of very blue galaxies (over 150000 per square deg per mag) ; gravitational lens images show that the redshift of galaxies fainter than mag 24 is in the range 1-3 (Tyson, Nature, 334, 294, 1988 ; Tyson and Seitzer, ApJ 335, 552, 1988 ; Tyson et al., ApJ, 349, L1, 1990 ; Guhathakurka et al., ApJ, 346, 773, 1989 and $357, \mathrm{L9}, 1990)$. 
A review of the methods of separation of the extragalactic background light from other much stronger components of the light of the night sky has been presented by Mattila (GEBR, 257). New results from the dark cloud method, in connection with improved observing techniques, allow to conclude that : 1) the method of using two parallel telescopes with simultaneous integration times and identical color filters is effective for the elimination of airglow fluctuations ; 2) it provides a very good accuracy for measuring the faint extended brightness of dark nebulae $(350-580 \mathrm{~nm}) ; 3)$ the combination of these optical results with the IRAS data provides information on the scattering and absorption properties of the grains ; 4) the extragalactic background light has a value of $\approx 3 S_{10}(v)$ at $400 \mathrm{~nm}$ (Mattila et al., GEBR, 99 and 461 ; A \& A, 184, 269, 1987 ; Morgan QJRAS, 28, 328, 1988).

\section{Future plans}

Many exciting projects will, hopefully, be carried out through this decade. The COBE and ISO satellites for the infrared domain are well known. COBE is already providing fascinating new results; ISO will have, in 1993, remarkable capabilities to disentangle the overlaying background components and will offer a large guest observer programme, for which preparations are to be made right now (Lemke, GEBR, 429).

In the extreme ultraviolet background domain, observations should be obtained from the wide field camera on board the ROSAT satellite (Harris et al., GEBR, 463). Also, in the far ultraviolet spectral range, structures will be imaged from the FAUST telescope during the ATLAS 1 shuttle mission (Lampton et al., GEBR,449) to be launched in 1991 ; and imaging spectrography is anticipated on the Spartan 281 shuttle mission (Carruthers et al., GEBR, 459), to be launched in $1992 \ldots$ 